\title{
Immunoperoxidase labelled antibody staining in differential diagnosis of central nervous system haemangioblastomas and central nervous system metastases of renal carcinomas
}

\author{
SUSAN M ANDREW, E GRADWELL \\ From the Department of Pathology, Whiston Hospital, Prescot, Merseyside
}

SUMMARY The problem of the differential diagnosis both of central nervous system haemangioblastomas and metastases of renal clear cell carcinomas was investigated by immunoperoxidase labelled antibody staining of five cases each of haemangioblastoma and metastatic renal carcinoma. Staining using anticarcinoembryonic antigen (CEA), antikeratin, antifactor VIII related antigen, and antibrush border was unhelpful. All five of the haemangioblastomas were negative and all five of the metastases were positive for epithelial membrane antigen (EMA), as defined by immunoperoxidase staining. This shows that commercially available anti-EMA monoclonal antibody is useful in distinguishing the two pathological entities.

Haemangioblastomas of the central nervous system occur most often in the cerebellum, occasionally in the spinal cord, and less commonly in the cerebrum. ${ }^{1}$ Histologically, they are vascular tumours with large pale stromal cells. The histogenesis of the stromal cell remains controversial. One recent ultrastructural study $^{2}$ claimed that Weibel-Palade bodies are present in their cytoplasm, indicating an endothelial derivation; another recent study ${ }^{3}$ has claimed that neurosecretory granules are present, indicating a neuroectodermal origin.

In the general laboratory these tumours present a diagnostic problem in that they are often histologically indistinguishable from central nervous system (CNS) metastases of renal clear cell carcinomas, as both entities are highly vascular with stromal clear cells, as seen by haematoxylin and eosin and reticulin stains, and both may show positivity in the stromal cells when stained with periodic Schiff reagent and variable positivity when stained for fat. ${ }^{1}$ This difficulty is occasionally compounded by the coexistence of haemangioblastomas and renal carcinomas in patients with the von Hippel-Lindau syndrome. We used a range of immunoperoxidase labelled antibodies to address this problem of differential diagnosis of the two entities.

Accepted for publication 13 March 1986

\section{Material and methods}

The material was obtained from the files of Whiston Hospital pathology department and Walton Hospital neuropathology department in the form of paraffin embedded blocks; the tissue had been primarily fixed in buffered formalin. The tissue consisted of surgical biopsy specimens from five cases of CNS haemangioblastoma and five cases of metastases to the CNS from renal carcinomas. The diagnoses were arrived at by a combination of careful clinical assessment at time of presentation, histological appearance of the lesion at light microscopy, and clinical follow up of between one and two years. Some of the cases had been diagnosed originally as haemangioblastomas, but the diagnosis had been changed after the clinical discovery of a primary renal carcinoma with or without further metastatic disease. Table 1 summarises the age and sex of the patients and the site of the tumours.

The tissue was treated in the same way as the surgical material passing through our laboratory. Paraffin sections were cut $(4 \mu \mathrm{m})$, dried at $37^{\circ} \mathrm{C}$ for an hour, and then fixed on to the slides by putting them into the $56^{\circ} \mathrm{C}$ oven for 10 minutes. The antibodies were applied to the slides according to the kit instructions, and in the case of the antibrush border, according to the description given by RC Nairn et al. ${ }^{4}$ The antibodies used were antifactor VIII related 
Table 1 Patient details

\begin{tabular}{clll}
\hline Case No & Sex & Age (years) & $\begin{array}{l}\text { Site of lesion as stated on histology } \\
\text { request form }\end{array}$ \\
\hline 1 & M & 45 & Right parietal \\
2 & M & 70 & Left occipital \\
3 & F & 73 & Right cerebellum \\
4 & M & 70 & Spinal cord T11 \\
5 & F & 43 & Right cerebello-pontine angle \\
6 & M & 38 & Right cerebellum \\
7 & F & 40 & Right cerebellum \\
8 & M & 55 & Left cerebellum \\
9 & M & 62 & Right cerebellum \\
10 & M & 22 & Left cerebellum \\
\hline
\end{tabular}

antigen (polyclonal Ortho), antikeratin (polyclonal, Ortho), anti-CEA (polyclonal Ortho), anti-EMA (monoclonal, Dako), and antibrush border (prepared by Dr TJ Matthews, Southampton University).

The staining procedure was controlled negatively by omitting the antibody, and positively by using skin for antikeratin, normal breast for anti-EMA, an adenocarcinoma of colon for anti-CEA, normal kidney for antibrush border, and by observing blood vessel endothelium in the section itself for antifactor VIII.

\section{Results}

Table 2 summarises the results. The anti-CEA was negative in all sections. The antifactor VIII related antigen was equivocally positive in the cytoplasm of stromal cells in two haemangioblastomas and clearly positive in the blood vessel endothelium of all sections examined: we could detect no consistent difference in vascular pattern, as shown by the factor VIII related antigen staining between the two groups. The antikeratin showed granular positivity similar to that seen in normal skin appendages in clear cell cytoplasm in two of the renal carcinomas and equivocal positivity in a third. The antibrush border was difficult to interpret because of high background staining but showed coarse granular positivity in the cytoplasm of the clear cells of one of the metastatic renal carcinomas.
The anti-EMA showed unequivocal positivity around the edges of clear cells in four of the renal carcinomas and patchy weak positivity in the fifth. The haemangioblastomas were all clearly negative for EMA.

\section{Discussion}

The problem of the differential diagnosis of metastatic renal carcinoma and primary haemangioblastoma may, in some cases, be resolved by careful review of the history, radiological findings, histological findings in paraffin embedded material, and, if available, the electron microscopic findings. There remain a certain number of cases, however, in which there is no haematuria, no radiological evidence of renal carcinoma, no distinctive histological features, and no evidence of epithelial differentiation on electron microscopy. Such cases must be primary haemangioblastomas and have to be followed up in the clinic. There does, however, remain some doubt, which the use of immunoperoxidase labelled antiEMA may simply, quickly, and relatively inexpensively help to dispel.

A distinct pattern of the blood vessels of haemangioblastomas, as shown by antifactor VIII related antigen staining of endothelium, has been reported. ${ }^{5}$ We did not find this a helpful way of distinguishing between the haemangioblastomas and the metastases.

The antikeratin staining, although present in two of the metastases, was not reliable enough to be recommended as a way of distinguishing between the two groups. The antibrush border antibody was not useful in this preparation, nor is it commercially available at the moment, so for current diagnostic problems it is not helpful; if a commercial preparation does become available, however, it would be worthwhile further evaluating its use in this diagnostic problem.

The most useful stain was the monoclonal antiEMA. Fleming $e t a^{6}$ found that EMA was present in 28 of 30 renal clear cell carcinomas that they exam-

Table 2 Summary of results of immunoperoxidase staining of stromal cells in two groups of cases

\begin{tabular}{|c|c|c|c|c|c|c|}
\hline Case No & Diagnosis & $\begin{array}{l}\text { Epithelial } \\
\text { membrane antigen }\end{array}$ & Keratin & Brush border & Factor VIII & Carcinoembryonic antigen \\
\hline $\begin{array}{l}1 \\
2 \\
3 \\
4 \\
5\end{array}$ & Metastatic renal carcinoma & $\begin{array}{l}+ \\
+ \\
+ \\
+ \\
\pm\end{array}$ & $\begin{array}{l}+ \\
\pm \\
\pm \\
+\end{array}$ & $\begin{array}{l}\overline{+} \\
\overline{-} \\
\overline{-}\end{array}$ & $\begin{array}{l}\overline{-} \\
\overline{-} \\
\overline{-}\end{array}$ & $\begin{array}{l}\overline{-} \\
\overline{-} \\
\overline{-}\end{array}$ \\
\hline $\begin{array}{r}6 \\
7 \\
8 \\
9 \\
10\end{array}$ & Haemangioblastoma & $\begin{array}{l}- \\
\overline{-} \\
\overline{-}\end{array}$ & $\begin{array}{l}- \\
\overline{-} \\
\overline{-}\end{array}$ & $\begin{array}{l}- \\
\overline{-} \\
- \\
-\end{array}$ & $\begin{array}{l} \pm \\
- \\
\pm \\
-\end{array}$ & $\begin{array}{l}\overline{-} \\
\overline{-} \\
\overline{-}\end{array}$ \\
\hline
\end{tabular}


ined. We found that it was present in all five renal carcinoma metastases and completely absent in all five haemangioblastomas.

The pattern of staining by the anti-EMA was specific in that it stained only the edges of the stromal cells of the renal carcinoma metastases. This specificity is helped by this particular antibody's monoclonality. The lack of specificity in the staining by the antikeratin antibody was probably due to its polyclonality, and if we had had a monoclonal anticytokeratin that worked on paraffin embedded material it may have been more useful in distinguishing the two lesions.

Despite the controversy over the histogenesis of the stromal cell of the haemangioblastoma no recent study has suggested that it is epithelially derived. Whether it is derived from endothelium or neuroectoderm the haemangioblastoma is not expected to express epithelial markers. This expectation was borne out by this study.

We believe that immunoperoxidase staining of paraffin embedded material using monoclonal antiEMA can make a helpful contribution to the differential diagnosis of haemangioblastomas and metastases of renal clear cell carcinomas in the central nervous system.

We thank Drs P Buxton and JV Diengdon of the
Department of Neuropathology, Walton Hospital, Merseyside, for sections and advice; Dr TJ Matthews, department of pathology, Southampton University Medical School, for the antibrush border antibody; Mrs PM Gurney for technical help and Miss AM Taylor for typing the manuscript.

\section{References}

${ }^{1}$ Rubinstein LJ. Tumours of the central nervous system. In: Atlas of tumour pathology. 2nd series fascicle 6. Washington, DC: Armed Forces Institute of Pathology, 1972:235-40.

${ }^{2}$ Khang-Loon $\mathrm{H}$. Ultrastructure of cerebellar capillary haemangioblastoma I. Weibel-Palade bodies and stromal cell histiogenesis. $J$ Neurol 1984;43:592-608.

${ }^{3}$ Ismail SM, Jasani B, Cole G. Histogenesis of haemangioblastomas: an immunochemical and ultrastructural study in a case of von Hippel-Lindau syndrome. J Clin Pathol 1985;38:417-21.

${ }^{4}$ Nairn RC, Richmond HG, MacEnteggart MG, Fothergill JE. Immunological differences between normal and malignant cells. Br Med J 1960;1335-9.

${ }^{5}$ Epstein JI, White CL, Mendelsohn G. Factor VIII related antigen and glial fibrillary acid protein immunoreactivity in the differential diagnosis of central nervous system haemangioblastomas. Am J Clin Pathol 1984;81:285-92.

${ }^{6}$ Fleming S, Lindop GBM, Gibson AAM. The distribution of epithelial membrane antigen in the kidney and its tumours. Histopathology 1985;9:729-39.

Requests for reprints to: Dr SM Andrew, Registrar in Histopathology, Whiston Hospital, Prescot, Merseyside L35 5DR, England. 